\section{A CALORIMETER FOR DETERMINING THE RELATION BETWEEN HEAT-PRODUCTION AND MUSCULAR WORK.}

$\mathbf{N}$ the physiological laboratory of the University of

Sheffield a calorimeter has been erected on the model of Atwater and Benedict's calorimeter, now carried by Prof. Benedict to a great state of perfection in the Nutrition Laboratory at Boston, U.S.A. The Sheffield copy is not at present a complete one, and its limitations are best detailed by a brief description of the problem in the solution of which it is hoped immediately to play some part. This problem is the nature of the physicochemical process underlying the phenomenon of muscular contraction, upon which some work has been in progress in this laboratory already along somewhat different lines.

A precise statement of the relation existing between muscular work and heat production is a. necessity for the final solution of this question. Even before precision can be hoped for, additional information may be of much value in deciding the comparative value of different lines of attack. Thus, it having been already shown that the energy liberated is entirely to be assigned to the combustion of food, the question arises as to whether the contraction is the direct consequence of the combustion which rakes place at the same time, or as to whether it is not rather the consequence of some preceding combustion. In the latter case there would be nothing remarkable in the proposition that the combustion responsible was in the main an occurrence taking place during a preceding contraction. Eacin contraction might, so to speak, vitid up a spring for release in the next contraction. The usual view is that contraction and the combustion responsible for it are concomitant; the view of the Sheffield laboratory is that they are not. The second point of view can be at once supported by the well-known fact that fatigued muscle is apparently a more economical heat engine than muscle in good condition, giving off less waste heat for an equal amount of external work done. Such a fact loses all peculiarity if it is considered merely as a failure on the part of the fatigued muscle to provide for succeeding contractions. It is in the hope of collecting further data of this kind that the calorimeter has been constructed. The immediate necessities, therefore, are a caiorimeter within which prolonged muscular work can be performed, and from which a complete statement of the amount of work done and heat given off can be obtained. Benedict's original instrument offers much more information than this, since it is a complete respiration calorimeter, providing by its use data for a perfect balance-sheet both of chemical material and energy. It is hoped to raise the Sheffield apparatus to the same level of perfection, so that it may be placed at the disposal of investigations of a more general kind when additional financial aid has been obtained.

The calorimeter has been constructed by Messrs. George, of Birmingham, who have followed the detailed instructions provided by Benedict in the publications of the Carnegie Institution, Washington. At one stage of con struction it was examined by Prof. Benedict, and then met with his approval. He was kind enough to make important suggestions, which have since been carried out.

A small room, 8 feet by 6 feet by 5 feet, has been built of copper sheets carefully soldered together and stiffened by an external wooden framework, to which they are fixed. On the outer surface of the framework zinc sheets have been fastened, forming a second metal covering

The double-walled metal box so formed rests on rails within a double-walled wooden house enclosing it. Thus the walls, copper, zinc, wood, and wood separate three layers NO. 20I8, VOL. 78$]$

of air external to the chamber. The accessory arrangements of the calorimeter render it possible to maintain the zinc sheath and the two outer layers of air at the same temperature as that of the copper box, whatever that may be. Heat is thus prevented from escaping from the walls of the copper chamber. This end is secured by heating and cooling apparatus in the two outer air spaces, resistance wires and cold-water pipes. The degree to which the heating and cooling mechanism is resorted to in each of these spaces is determined by the observation of electrical currents from one hundred and fifty sets of thermocouples arranged in two series, a series indicating differences of temperature between the copper and zinc sheaths, another indicating differences between the two outer air spaces. Each series is divided into zones, so that the state of the roof, the floor, or each of three zones in the side walls can be separately observed. The heating and cooling mechanism is similarly subdivided. The observer seated outside the calorimeter can rapidly test each segment of the calorimeter in turn, and rapidly balance any difference found by adjustments of rheostats admitting more or less current to the heating wires, and 
provide and remove fear. of defects in this direction. When to this is added the risk introduced by every screw passing through the copper, such as, for example, those necessary for the fixation of the bed, the balance beams from which the radiator system is suspended, \&c., there is at present fear. enough. It was in indicating points such as this that Prof. Benedict's visit was of so much value.

The heat produced within the copper box, carefully prevented from escaping from its surface in the manner described, is carried out in a stream of water constantly driven through the radiator system. The quantity of water passing is varied with the necessities of the moment, and is necessarily much greater when the subject is at work on the ergometer. The water passing is weighed on exit in a meter, and its temperature on entrance and exit observed. The former is practically constant, the latter kept as constant as possible by variation in the rate of water flow. These data form the main items in the statement of heat produced, though other important items, such as the amount of water condensing on the radiator pipes within the chamber, the water evaporated and carried through with the air leaving the chamber, are duly considered and provided for. Thermocouples are placed in the tubes through which the air finds entrance and exit, in quantity 75 litres per minute, so as to ensure the detection of any difference of temperature. Any difference found is corrected by increased heating of the entering air.

In Benedict's calorimeter the air is driven from the chamber through a closed system of tubes back to the chamber again. In this system are interpolated sulphuric acid and soda lime absorbers of a necessarily very large size, determined by the large mass of air in motion per unit of time. The oxygen consumed is made good by admission of oxygen from a cylinder of the compressed gas. In the Sheffield apparatus this will not, at first at least, be attempted. Dried air heated and passer into the chamber will be driven out through a sulphuric acid absorber, no attempt being made to do more than take a determination of the water.

\section{RECENT RESEARCHES IN THE STRUCTURE} OF THE UNIVERSE. ${ }^{1}$

I CONSIDER it an uncommon privilege to lecture on the structure of the universe in the country of the Herschels. Even now their celebrated gauges are unrivalled, and they still form one of the important elements on which any theory of the stellar system must be based. It is well known that the plan of these gauges consisted in directing the telescope successively to different points all over the sky, and simply counting the number of stars visible in the freld.

There is one fact clearly brought out by these gauges to which I must direct your attention. It is that in the outward appearance of our nightly sky, as seen with the telescope, there is a great regularity. In the Milky Way, that belt which we see with the naked eye encircling the whole of the firmament nearly along a great circle, the number of stars, as seen in Herschel's 20 -feet reflector, is enormous. On both sides this apparent croivding of the stars diminishes very gradually and regularly until, near the poles of the Milky Way, we come to the poorest parts of the sky.

Let us look at this phenomenon somewhat more closely. If we direct our telescope first towards the part of the Milky Way near Sirius, and if from there we gradually work up towards the North Pole of the Milky Way in the constellation called the Hair of Berenice, we shall clearly perceive this gradual and regular change in the number of stars. Now if we repeat the same process, beginning from some other point of the Milky Way, say in Cassiopeia or the Southern Cross, we shail find that not only is there a similar gradual change, but we shall approximately go through the same changes.

At the same distance from the Milky Way we shall find, approximately, the same number of stars in the field of the telescope. Put in other words, the richness of stars

1 Discourse delivered at tue Royal Institution on Friday, May 22, by Prof. J. C. Kapteyn.

NO. 2018 , VCL. 781 varies regularly with the galactic latitude; it varies relatively little with the galactic longitude.

Imitating most of the investigators of the stellar system, we will therefore disregard the longitude and keep in view only the changes with the galactic latitude. In reality this comes to being satisfied with a first approximation. For, in reality, there are differences in the different longitudes, especially in the Milky Way itself. But even here the differences are not so great as seems commonly to be supposed. There is every reason to believe, therefore, that our approximation will be already a toler bly close one.

\section{Real Structure.}

Meanwhile, what the Herschel gauges teach us is only relative to the outward appearance of the sky. What is the real structure of the stellar world? If we see so many stars in the field, with the telescope directed to the Milky Way, is it because they are really more closely crowded there, as Struve thinks, or is the view of the older Herschel correct, who imagined that the greater richness is simply a consequence of the fact that we are looking in deeper layers of stars; that our universe is more extensive in the Milky Way than it is in other directions?

Imagine that we could actually travel through space. For instance, imagine that first we travel in the direction of the constellation Cassiopeia. If we travel with the velocity of light, not so very many years would pass before we get near to some star. Proceeding on our journey for many, many more years, always straight on, we will pass more stars by and by. How will these stars look thus viewed from a moderate distance-say, from a distance as that of the sun? Will they all be found to be of equal luminosity, as Struve practically assumed? And in this case are they as luminous as our sun, or more so, or less so? Or are they unequal? If so, how many of them are brighter than our sun, how many fainter? Or, to be more particular, how many per cent. of the stars are ro, too, rooo, \&c., times more luminous than our sun? How many are equal to the sun, or ro, roo times fainter? In a few words: What is the nature of the mixture? or, lastly, what is the mixture law of the system of the stars?

Furthermore, in travelling on, shall we find the stars in reality equally thickly, or rather thinly, crowded everywhere? Or shall we find that after a certain time, which may be many centuries, they begin to thin out, as a first warning of an approaching limit of the system? Is there really such a limit, which, once passed, leads us into abysses of void space?

Herschel thought there was such a limit, and even imagined that his big telescope penetrated to that limit; that is, he assumed that his telescope made even the remotest stars visible. On this supposition is based his celebrated disc theory of the system.

Again, we may condense these questions in this single query: How does the crowding of the stars, or the stardensity, that is, the number of stars in any determined volume (let us say in a cubic light century), vary with the distance from our solar system?

But there is more. We supposed that our journey went straight on in the direction of Cassiopeia, which is in the Milky Way. What if our journey is directed to the Pleiades, which are at some distance from that belt, or to the Northern Crown, which is still further, or to the Hair of Berenice, which is furthest of all from the Milky Way? For different regions equally distant from the galaxy we have seen that outward appearances are the same. We may admit, with much probasility, that in space, too, we would find little difference. Summing up, the problem of the strudture of the stellar system in a first approximation comes to this :-

To determine, separately for regions of different galactic latitude, in which way the star-density and the mixture vary with the distance from the solar system.

I think that there is well-founded hope that, even perhaps within a few years, sufficient materials will be forthcoming which will allow us to attack the problem to this degree of generality, with a fair chance of success. At the present moment, however, our data are yet too scanty for the purpose. Still, they will be sufficient for the derivation of what must be in some sort ajerage con- 